Estudio de los daños estructurales tras la inmovilización espermática previa a la ICSI en sujetos teratozoospérmicos

Gómez-Torres $\mathrm{MJ}^{1,{ }^{*}}$; García EM ${ }^{1,2}$; Guerrero $\mathrm{J}^{2}$, Girela $\mathrm{JL}^{1}$; Ten $\mathrm{J}^{1,2}$; Bernabeu $\mathrm{R}^{2}$; De Juan J.

${ }^{1}$ Department of Biotechnology, University of Alicante, Campus de San Vicente del Raspeig, San Vicente del Raspeig 03080, Alicante, Spain. ${ }^{2}$ Department of Reproductive Biology and Medicine, Instituto Bernabeu, Alicante 03016, Spain

*Correspondencia: María José Gómez Torres. Departamento de Biotecnología, Universidad de Alicante, Campus de San Vicente del Raspeig, San Vicente del Raspeig 03080. Tfno: +34 96 5903999. Correo electrónico: mjose.gomez@ua.es. 


\title{
Estudio de los daños estructurales tras la inmovilización espermática previa a la ICSI en sujetos teratozoospérmicos
}

\author{
Resumen
}

Objetivo: Describir y comparar las alteraciones ultraestructurales que puede provocar la inmovilización espermática, previa a la ICSI, así como los daños en el DNA y estado del acrosoma en espermatozoides de sujetos teratozoospérmicos y normozoospérmicos,

Material y métodos: Se utilizaron 15 muestras seminales procedentes de donantes normozoospérmicos y 20 muestras de pacientes teratozoospérmicos del Instituto Bernabeu de Alicante. Para el estudio el Microscopia Electrónica de Transmisión se utilizaron ovocitos humanos como receptáculo de los espermatozoides. La fragmentación del ADN se valoró mediante la técnica TUNEL y el estado del acrosoma utilizando la lectina Pisum sativum conjugada con FITC. El análisis estadístico entre los diferentes grupos se realizó mediante un test ANOVA.

Resultados: Los resultados mostraron que, tras la inmovilización, tanto los espermatozoides procedentes de sujetos normozoospérmicos y teratozoospérmicos sufrieron las mismas alteraciones ultraestructurales a nivel de la membrana plasmática y acrosomal. En cambio, no se observaron daños a nivel del núcleo. Tras valorar la fragmentación de ADN mediante TUNEL en sujetos normozoospérmicos y teratozoospérmicos, observamos que en los espermatozoides inmovilizados el porcentaje de espermatozoides con ADN fragmentado era similar en ambos grupos. En cambio, el porcentaje de espermatozoides reaccionados fue significativamente más elevado en los espermatozoides inmovilizados procedentes de sujetos teratozoospérmicos que en el grupo control. 
Conclusión: Los resultados de este estudio sugieren que los daños provocados por la inmovilización, previa a la ICSI, en espermatozoides procedentes de sujetos normozoospérmicos y teratozoospérmicos son independientes de la morfología espermática.

Palabras clave: ICSI, inmovilización, teratozoospermia, ultraestructura, reacción acrosómica, fragmentación DNA. 
Tittle:

\section{Study of structural damage after sperm immobilization prior to ICSI in teratozoospermic males}

Objective: To describe and to compare the ultrastructural alterations, DNA fragmentation and acrosome status that can cause sperm immobilization prior to the ICSI, in samples from normozoospermic and teratozoospermic males.

\section{Material and methods}

For this study fifteen sperm samples from normozoospermic consenting donors and twenty samples from teratozoospermic males, submitted for assisted reproduction at Instituto Bernabeu of Alicante, were used. To achieve the assessment of the ultrastructural alterations induced by immobilization, human oocytes were used as containers for spermatozoa, and then were observed by Transmission Electron Microscopy (TEM). DNA fragmentation was assessed by TUNEL and acrosome status using conjugated fluorescein isothiocyanate-labeled Pisum sativum agglutinin (FITC-PSA). The control group was sperm without manipulation. Statistical analysis between groups was performed by ANOVA.

\section{Results:}

The results showed that immobilized spermatozoa presented the same damage in plasma membrane and acrosomal membrane, both in the samples from normozoospermic and teratozoospermic subjects. Moreover, no changes were observed at nucleus. 
After assessing DNA fragmentation by TUNEL technique in the cells from normozoospermic and teratozoospermic patients, we observed that the percentage of spermatozoa with DNA fragmentation didn't increase in immobilized group compared with control group. However, the percentage of acrosome reacted sperm was significantly greater in immobilized spermatozoa than in control group, in teratozoospermic males.

Conclusion: The results of this study suggest that the damage caused by immobilization prior to ICSI in the spermatozoa from teratozoospermic and normozoospermic males is independent of sperm morphology.

Key words: ICSI, immobilization, teratozoospermic, ultrastructure, acrosome reaction, DNA fragmentation. 


\section{INTRODUCCIÓN}

La esterilidad afecta al $10-15 \%$ de las parejas ${ }^{1}$, siendo el factor masculino responsable en aproximadamente el $50 \%$ de los $\operatorname{casos}^{2}$. La incorporación de la microinyección intracitoplasmática de espermatozoides (ICSI) $)^{3,4}$ como técnica de rutina, supuso una enorme revolución en el campo de la medicina reproductiva, al solucionar la mayor parte de causas de esterilidad debidas a un factor masculino.

Actualmente, la ICSI es la técnica de reproducción asistida por excelencia, empleada en la mayoría de los tratamientos de infertilidad por todo el mundo. No obstante, y a pesar de que es una técnica de reproducción más invasiva que la fecundación in vitro convencional y que la micromanipulación de los gametos podría inducir determinados riesgos genéticos y epigenéticos ${ }^{5}$, su uso se está efectuando en muchas clínicas de reproducción asistida de manera indiscriminada. Entre los años 1999 y 2004 el empleo de la ICSI se incrementó de forma dramática, pasando de un $11.0 \%$ a un $57.5 \%$, mientras que el porcentaje de parejas con esterilidad atribuídas a un factor masculino se mantuvo estable ${ }^{6}$.

La inmovilización del espermatozoide, previa a la microinyección intracitoplasmática de espermatozoides o ICSI, es un paso esencial para que ocurra la fecundación ${ }^{7,8}$, ya que permite la desestabilización y permeabilización de la membrana plasmática ${ }^{9,10}$. Algunos autores han observado que la inmovilización espermática y la permeabilización de la membrana plasmática mejora los porcentajes de fecundación ${ }^{11,12}$, ya que esta manipulación facilita la descondensación nuclear tras la inyección del espermatozoide ${ }^{9}$, así como la liberación del hipotético factor espermático soluble (SOAF) que induce la activación del ovocito ${ }^{13}$. La inmovilización espermática se realiza, generalmente, mediante procedimientos mecánicos o físicos. Sin embargo, la técnica más utilizada en ICSI suele ser la inmovilización mecánica conocida como convencional o squeezing ${ }^{11,14,15,16}$ que consiste en dar un golpe, con la pipeta de microinyección, en el flagelo del 
espermatozoide. En este sentido, diversos estudios sugieren que la inmovilización mecánica es una técnica invasiva, ya que contacta directamente con el espermatozoide aplicando la fuerza sobre la pieza intermedia, lo que podría generar importantes alteraciones a nivel celular. Nosotros hemos descrito, algunas de estas alteraciones producidas en los espermatozoides humanos, inmovilizados mediante la técnica convencional o squeezing, utilizando la microscopía electrónica de barrido y la microscopia confocal ${ }^{17}$.

Está demostrado que en casos en los que existe un factor masculino severo, azoospermia con espermatozoides de biopsia testicular, criptozoospermia, oligozoospermia, astenozoospermia, teratozoospermia, con la ICSI se obtienen muy buenos resultados ${ }^{18}$. En todas estas patologías, la inmovilización de los espermatozoides, previa a la ICSI, se realiza utilizando la misma metodología, sin tener en cuenta las características citomorfológicas de las muestras. En base a esto el objetivo principal de este trabajo fue describir los daños ultraestructurales, fragmentación del DNA y estado del acrosoma que la inmovilización espermática, previa a la ICSI, provoca en los espermatozoides procedentes de sujetos teratozoospérmicos y normozoospérmicos. Pero uno de los inconvenientes que nos encontramos a la hora de estudiar pocas células con el Microscopio Electrónico de Transmisión (MET), es la forma de visualizarlos. Para ello y de forma novedosa, en este trabajo empleamos ovocitos humanos como receptáculos de los espermatozoides inmovilizados para asegurarnos su visualización al MET.

\section{MATERIALY MÉTODOS}

Las muestras de semen que se incluyeron en el estudio, se obtuvieron de 15 donantes voluntarios, normozoospérmicos y de 20 pacientes teratozoospérmicos, del Instituto Bernabeu (Alicante, España). Las muestras fueron obtenidas tras 3-5 días de abstinencia 
y el diagnóstico de las muestras fue evaluado siguiendo las normas de la OMS ${ }^{1}$. Todos los pacientes firmaron previamente el consentimiento informado.

Para cada muestra se establecieron dos grupos con 200 espermatozoides móviles, seleccionados mediante la técnica de Swim-up y ajustados a una concentración final de $3 \times 10^{6}$ espermatozoides $/ \mathrm{mL}$. Los espermatozoides fueron inmovilizados mediante squeezing en su pieza principal del flagelo (Grupo Inmovilizados). El Grupo Control, fueron espermatozoides móviles, a los que no se les había manipulado.

Para poder visualizar los espermatozoides en el MET, se usaron como receptáculos, ovocitos humanos, inmaduros o de fallos de fecundación in vitro (los cuales no presentaron evidencias de pronúcleos después de las 48 horas de la fecundación). Los ovocitos se obtuvieron de mujeres incluidas en el programa de reproducción asistida del Instituto Bernabeu de Alicante. Todas las pacientes firmaron previamente el consentimiento informado.

Aproximadamente 200 espermatozoides de cada muestra, previamente inmovilizados, fueron aspirados en una pipeta de ICSI y microinyectados en el espacio perivitelino de los ovocitos. El mismo número de espermatozoides del grupo control fueron inyectados en los ovocitos de la misma forma, pero sin la manipulación previa. Dicho procedimiento puede observarse en la Figura 1.

Después de la inyección, los ovocitos fueron fijados al $2 \%$ de glutaraldehído e incluidos en resina LRWhite. Los bloques fueron cortados en un ultramicrotomo LEICA ULTRACUT UCT® (Leica Mickrosysteme GmbH, Viena, Austria), con cuchilla de diamante. Las rejillas fueron teñidas con acetato de uranilo y examinadas con MET (Philips TECNAI 12).

El estado de fragmentación del DNA espermático fue evaluado mediante la técnica TUNEL (In Situ Cell Death Detection Kit, Fluorescein, Roche Biochemicals, Mannheim, Germany). El porcentaje de espermatozoides con fragmentación de DNA se determinó 
usando un microscopio confocal (Leica DM IRBE2), empleando un objetivo con aceite de inmersión a 1000X. Cada muestra fue analizada por duplicado y en cada experimento se realizaron controles positivos y negativos. Los resultados se expresaron como porcentajes (\%) de espermatozoides con fragmentación de DNA.

Para la valoración de la reacción acrosómica, los espermatozoides fueron fijados y permeabilizados en metanol. A continuación se incubaron durante 30 minutos con la lectina Pisum sativum conjugada con FITC (PSA-FITC; Sigma Chemical Campany, St. Lowis, USA), tras realizar tres lavados en PBS, las preparaciones se montaron con el medio VectaShield (Vector Laboratories, Inc.; Burlingame, USA) y observadas con un microscopio confocal (Leica DM IRBE2). Se contaron 200 células en cada uno de los casos y se establecieron dos patrones, espermatozoides reaccionados y espermatozoides $\sin$ reaccionar $^{19}$.

Una vez comprobado que los resultados obtenidos se ajustaban a una distribución normal se realizó un Análisis de la Varianza ANOVA para comparar las posibles diferencias. Todos los análisis fueron realizados utilizando el paquete estadístico SPSS 15.0 (SPSS Inc., Chicago, IL, USA).

\section{RESULTADOS}

\section{Alteraciones ultraestructurales observadas observadas con MET}

De cada uno de los casos, se introdujeron entre 100-200 espermatozoides en el interior de los ovocitos, al procesarlos y visualizarlos al MET, la media de espermatozoides analizados fueron entre 50-60.

En el grupo control, tanto en sujetos normos como teratozoospérmicos, las células espermáticas no presentan alteraciones a nivel ultraestructural. Tal y como se muestra en la Figura 2a, la cabeza espermática de dichos gametos presentó la membrana plasmática 
íntegra, el acrosoma intacto, una envoltura nuclear normal y un núcleo sin fragmentación aparente. En las secciones donde pudimos visualizar la pieza intermedia, comprobamos que su estructura no presentaba ninguna alteración. De la misma forma, a nivel del axonema pudimos observar que la organización de los microtúbulos fue correcta, al igual que la disposición de la vaina fibrosa.

Las alteraciones ultraestructurales provocadas por la inmovilización, de los espermatozoides procedentes tanto de los sujetos normos como teratozoospérmicos, fueron las mismas. El $90 \%$ de las células estudiadas presentaron daños a nivel de la membrana plasmática así como de las membranas acrosomales (Figura $2 b$ y c). La mayoría de estos gametos presentaron acrosomas alterados. A nivel del núcleo, en la disposición de los microtúbulos y/o fibras densas, no se observaron alteraciones. Sin embargo, algunas células espermáticas sí mostraron una disposición anormal de las mitocondrias en la pieza intermedia, aunque el porcentaje de células con este tipo de alteración no fue significativo.

\section{Fragmentación del ADN}

Con el fin de comprobar a nivel molecular si la inmovilización provoca la fragmentación del ADN, empleamos la técnica TUNEL. Observamos que en el caso de los normozoospérmicos, el porcentaje de espermatozoides con ADN fragmentado fue del 2 '3\% en el grupo control y de 1 '2\% en el grupo de espermatozoides inmovilizados. Al realizar el análisis estadístico, no encontramos diferencias significativas entre ambos grupos. Sin embargo, en los sujetos teratozoospérmicos el grupo control mostró un porcentaje de espermatozoides con fragmentación de ADN significativamente superior al observado en el grupo de espermatozoides inmovilizados (5'9\% y $1 \%$ respectivamente; $\mathrm{p}<0 \times 001)$ 


\section{Valoración del estado del acrosoma}

En cuanto al acrosoma, en los sujetos con teratozoospermia, se observan diferencias significativas en el porcentaje de espermatozoides que habían sufrido reacción acrosómica entre el grupo control (2'4\%) y el grupo de espermatozoides inmovilizados (69'7\%). En estudios previos de nuestro grupo ${ }^{17}$, encontramos diferencias significativas entre el grupo control y el grupo de espermatozoides inmovilizados en sujetos normozoospérmicos (24\% y 95'5\% respectivamente).

\section{DISCUSION}

La inmovilización espermática previa a la ICSI es necesaria para poder incrementar de forma significativa, el porcentaje de fecundación ${ }^{8,20,12,21,10}$. Es importante conocer si los daños producidos tras la inmovilización espermática son más acusados en aquellas muestras que presenten alguna alteración citomorfológica, ya que actualmente este procedimiento se realiza utilizando la misma metodología en todos los casos. Entre las diferentes patologías espermáticas, decidimos incluir en este estudio las muestras teratozoospérmicas ya que parecen ser las muestras patológicas más vulnerables ${ }^{22}$. Estudios anteriores han mostrado que la inmovilización espermática puede causar alteraciones en las células espermáticas a nivel de la membrana plasmática, citoesqueleto y acrosoma en muestras normozoospérmicas ${ }^{23,17}$. En nuestro trabajo se compara de forma novedosa, las alteraciones a nivel ultraestructural que puede provocar la inmovilización previa a la ICSI en sujetos teratozoospérmicas frente a normozoospérmicos, concluyendo que los daños son similares en ambos grupos. 
Del estudio mediante microscopia electrónica, podemos concluir que uno de los orgánulos más afectados son los acrosomas. La mayoría de las células espermáticas a las que hemos sometido a inmovilización presentan el acrosoma alterado, lo cual se correlaciona perfectamente con los datos obtenidos de reacción acrosómica mediante el uso de la lectina PSA-FITC. Estos resultados confirman que la inmovilización induce la RA en los gametos de los sujetos teratozoospérmicos, pero si comparamos los resultados con los obtenidos por nuestro grupo en trabajos previos ${ }^{17}$, podemos ver que en el caso de los sujetos normozoospérmicos, la inmovilización induce la reacción acrosómica en un porcentaje mayor de células. Esto concuerda con resultados de otros autores, que encontraron una disminución del porcentaje de espermatozoides reaccionados, en presencia de zonas pelúcidas solubilizadas, al comparar sujetos normos y teratozoospermia severa ${ }^{24}$.

A nivel ultraestrucural, observamos que el proceso de inmovilización realizado previamente a la ICSI, no parece inducir alteraciones a nivel del núcleo espermático, en ninguna de las muestras analizadas. Sin embargo, mediante la técnica TUNEL, observamos que el porcentaje de espermatozoides con ADN fragmentado es mayor en el grupo control que en el grupo de inmovilizados, tanto en sujetos normozoospérmicos como teratozoospérmicos, siendo estas diferencias significativas en los sujetos con alteración de la morfología. Nuestros resultados del grupo control están en concordancia con los porcentajes mostrados previamente en otros estudios ${ }^{25,26,27,28}$. Aunque es conocido que el Swim-up selecciona los espermatozoides con mejor motilidad, morfológicamente normales y con menos daños a nivel del $\operatorname{ADN}^{29,30,31}$, en el caso de las muestras teratozoospérmicas, esta selección espermática, puede ser menos eficaz, y por ello encontramos en estos casos, diferencias significativas entre los espermatozoides del 
grupo control y los manipulados, en los que hemos realizado una segunda selección morfológica, por parte del micromanipulador, antes de realizar la inmovilización.

Por lo tanto, y coincidiendo con otros autores ${ }^{32}$, podemos afirmar que el proceso de inmovilización espermática previa a la ICSI no provoca daños aparentes a nivel nuclear en muestras normo ni teratozoospérmicas, ya que el porcentaje de espermatozoides con ADN fragmentado es inferior al obtenido en el grupo control.

Como conclusión, podemos decir que tanto en sujetos con parámetros seminales normales como en aquellos con alteración de la morfología espermática, la inmovilización realizada en los espermatozoides previa a la ICSI, no provoca alteraciones en la integridad del ADN, empelando la técnica TUNEL. Sin embargo este procedimiento, en todos los gametos estudiados, provoca una inducción mecánica de la reacción acrosómica, pudiendo este hecho favorecer la salida de factor(es) citosólico del espermatozoide encargados del activar al ovocito ${ }^{33}$. Nuestros resultados por tanto revelan que los daños provocados por la inmovilización, son independientes de las características seminales y además reafirman el éxito de la técnica ICSI en reproducción asistida.

\section{Financiación}

Este trabajo ha sido subvencionado por el proyecto de Investigación Emergente de la Universidad de Alicante (GRE08-P08) y el Vicerrectorado de Investigación de la Universidad de Alicante (VIGROB-137).

Bibliografía 
- World Health Organization (2010). WHO laboratory manual for the examination and processing of human semen (5th Edn). World Health Organization Press, Geneva.

- Huynh T, Mollard R, Trounson A. Selected genetic factor with male infertility. Hum Reprod Update 2007;8Supl:183-98.

- Palermo G, Joris H, Devroey P, Van Steirteghem AC. Pregnancies after intracytoplasmic injection of a single spermatozoon into an oocyte. Lancet 1992; 340: 17-8.

- Van Steirteghem AC, Nagy Z, Joris H, Liu J, Staessen C, Smitz J et al. High fertilization and implantation rates after intracytoplasmic sperm injection. Hum Reprod 1993;8Supl7:1061-6.

- Georgiou I, Syrrou M, Pardalidis N, Karakitsios K, MAntzavinos T, Giotitsas N et al. Genetic and epigenetic risks of intracytoplasmic sperm injection method. Asian J Androl 2006;8Supl6:643-73.

- Jain T, Gupta RS. Trends in the use of intracytoplasmic sperm injection in the United States. N Engl J Med 2007;357:251-7.

- Svalander P, Forsberg AS, Jakobsson AH, Wikland M. Factors of importance for the establishment of a successful program of intracytoplasmic sperm injection treatment for male infertility. Fertil Steril 1995;63:828-37.

- Bertin G, Lejeune B, Nijs M, Vandamme B, Schoysman R. Two essential steps for a successful intracytoplasmatic sperm injection: injection of immobilized spermatozoa alters rupture of the oolemma. Hum Reprod 1996;11:540-7.

- Dozortsev D, Rybouchkin A, De Sutter P, Dhont M. Sperm plasma membrane damage prior to introcytoplasmic sperm injection: a necessary condition for sperm nucleus descondensation. Hum Reprod 1995;10:2960-64.

- Palermo G, Schlegel PN, Colombero LT, Zaninovic N, Moy F, Rosenwaks Z. Aggressive sperm immobilization prior to intracytoplasmic sperm injection with immature spermatozoa improves fertilisation and pregnancy rates. Hum Reprod 1996;11:1023-9. 
- Palermo G, Joris H, Derde MP, Camus M, Devroey P, Van Steirteghem A. Sperm characteristics and outcome of human assisted fertilisation by subzonal insemination and intracytoplasmic sperm injection. Fertil Steril 1993; 59:826-35.

- Mangelschots K, Van Royen E, Joostens M, Eestermans W, Ryckaert G. ICSI and severe male-factor infertility: breaking the tail prior to injection. Hum Reprod 1995;10:484-6.

- Dozortsev D, Quian C, Ermilov A, Rybouchkin A, De Sutter P, Dhont M. Sperm associated oocyte activating factor is released from the spermatozoon within $30 \mathrm{~min}$ after injection as a result of the sperm-oocyte interaction. Hum Reprod 1997;12:27926.

- Sakkas D, Urner F, Bianchi PG, Bizarro D, Wagner D, Jaquenoud N et al. Sperm chromatin anomalies can influence descondensation after intracytoplasmic sperm injection. Hum Reprod 1996;11: 837-43.

- Dozortsev D, De Sutter P, Dhont M. Behaviour of spermatozoa in human oocytes displaying no or one pronucleus after intracytoplasmic sperm injection. Hum Reprod 1994;9:2139-44.

- Silber SJ, Nagy Z, Liu J, Tournaye H, Lissens W, Ferec C et al. The use of epididymal and testicular spermatozoa for intracytoplasmic sperm injection: the genetic implications for male infertility. Hum Reprod 1995;10:2031-43.

- Gómez-Torres MJ, Ten J, Girela JL, Romero J, Bernabeu R, De Juan J. Sperm immobilized before intracytoplasmic sperm injection undergo ultrastructural damage and acrosomal disruption. Fertil Steril 2007; 88:702-4.

- Brassesco Macazzaga A, Rovira Fontanals S, del Rio Bueno F, Cairo Doncos O, Monqaut A, Lafuente Varea R. ¿Es la inyección intracitoplasmática de espermatozoides morfológicamente seleccionados una técnica del futuro? Rev Int Androl 2010;8:57-9.

- Mendoza C, Carreras A, Moos j, Tesarik J. Distinction between true acrosome reaction and degenerative loss by one-step staining method using Pisum sativum agglutinin. J Reprod Fertil 1992;95:755-63. 
- Fishel S, Lisi F, Rinaldi L, Green S, Hunter A, Dowell K et al. Systematic examination of immobilizing spermatozoa before intracytoplasmic sperm injection in the human. Hum Reprod 1995;10:497-00.

- Van den Bergh M, Bertrand E, Biramane J, Englert Y. Importance of breaking a spermatozoon's tail before intracytoplasmic injection: a prospective randomised trial. Hum Reprod 1995;10:2819-20.

- Mehdi M, Khantouche L, Ajina M, Saad A. Detection of DNA fragmentation in human spermatozoa: correlation with semen parameters. Andrologia 2009;41:383-6.

- Takeuchi T, Colombero LT, Neri QV, Rosenwaks Z, Palermo GD. Does ICSI require acrosomal disruption? An structural study. Hum Reprod 2004;19:114-7.

- Bastiaan HS, Windt ML, Menkveld R, Kruger TF, Oehninger S, Franken DR. Relationship between zona pellucid-indiced acrosome reaction, sperm morphology, sperm-zona pellucid binding and in vitro fertilization. Fertil Steril 2003;79Supl1:49-5.

- Sun JG, Jurisicova A, Casper RF. Detection of deoxyribonucleic acid fragmentation in human sperm: correlation with fertilization in vitro. Biol Reprod 1997;56:602-7.

- Lopes S, Jurisicova A, Sun JG, Casper RF. Reactive oxygen species: potential cause for DNA fragmentation in human spermatozoa. Hum Reprod 1998;13:896-0.

- Muratori M, Piomboni P, Baldi E, Filimberti E, Pecchioli P, Moretti E et al. Functional and ultrastructural features DNA-fragmented human sperm. J Androl 2000;21:903-12.

- Muratori M, Maggi M, Spinelli S, Filimberti E, Forti G, Baldi E. Spontaneous DNA fragmentation in swim-up selected human spermatozoa during long time incubation. $\mathrm{J}$ Androl 2003;24:253-62.

- Oehninger S, Acosta R, Morshedi M, Philput C, Swanson RJ, Acosta AA. Relationship between morphology and motion characteristics of human spermatozoa in semen and in the swim-up sperm fractions. J Androl 1990;11:446-52. 
- Younglai EV, Holt D, Brown P, Jurisicova A, Casper RF. Sperm swim-up techniques and DNA fragmentation. Hum Reprod 2001;16Supl 9:1950-3.

- Piomboni P, Bruni E, Capitani S, Gambera L, Moretti E, La Marca A et al. Ultrastructural and DNA fragmentation analyses in swim-up selected human sperm. Arch Androl 2006; 52:51-9.

- Xu ZP, Sun HX, Hu YL, Zhang NY, Zhao X. Lasser-assisted immobilization causes no direct damage to sperm DNA. Zhonghua Nan Ke Xue 2007;13:216-8.

- Yanagida K, Katayose H, Hirata S, Yazawa H, Hayashi S, Sato A. Influence of sperm immobilization on onset of Ca2+ oscillations after ICSI. Hum Reprod 2001;16:148-52.

\section{LEYENDAS:}

Figura 1: Procedimiento experimental donde se ilustra la microinyección de los espermatozoides en el espacio perivitelino de los ovocitos humanos: A y B) Microfotografías de microscopía óptica de ovocito humano metafase I con espermatozoides en el espacio perivitelino. 50X y 100X. C) Microfotografía de 
microsccopía electrónica de transmisión de ovocito metafase I con espermatozoides en espacio perivitelino. 970X.
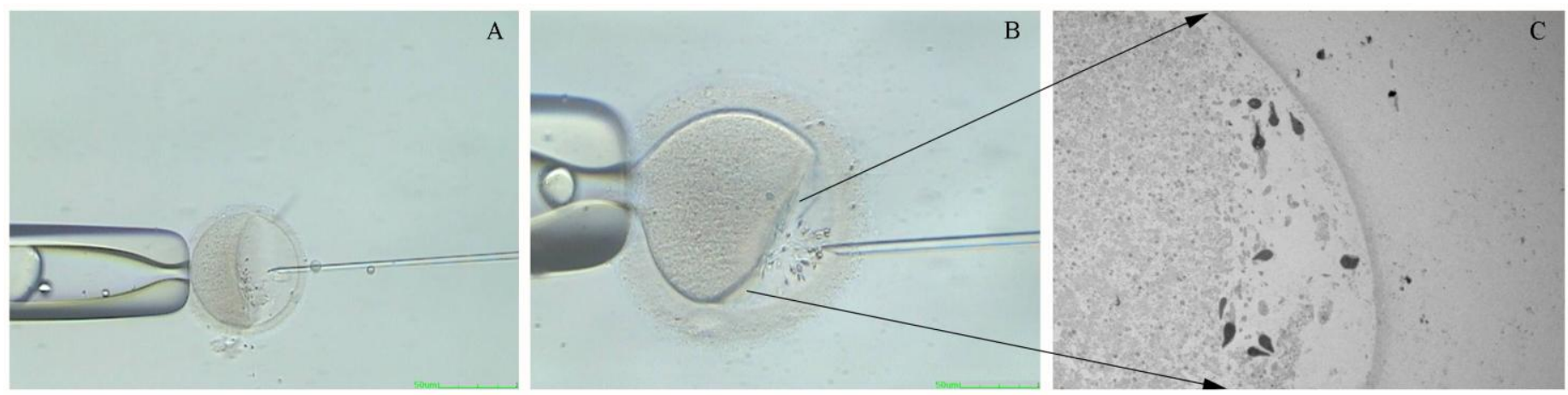

Figura 2: Microfotografías de microscopía electrónica de transmisión de espermatozoides humanos (A) cabeza morfológicamente normal. (B) y (C) cabezas con daño a nivel de la membrana plasmática y membranas acrosomales. 21.000X. 
Click here to download high resolution image
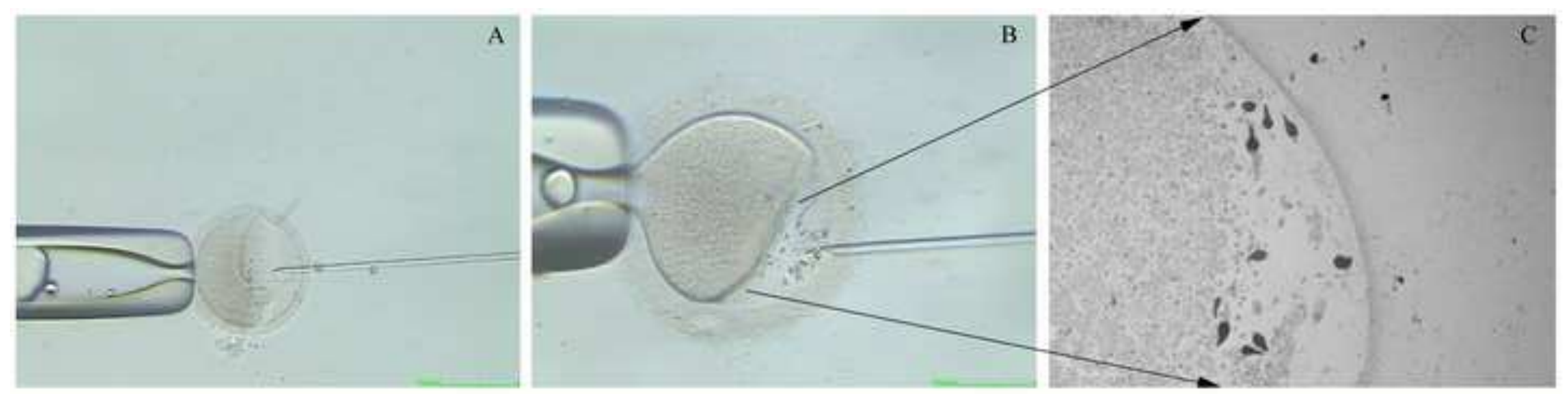


\section{Click here to download high resolution image}
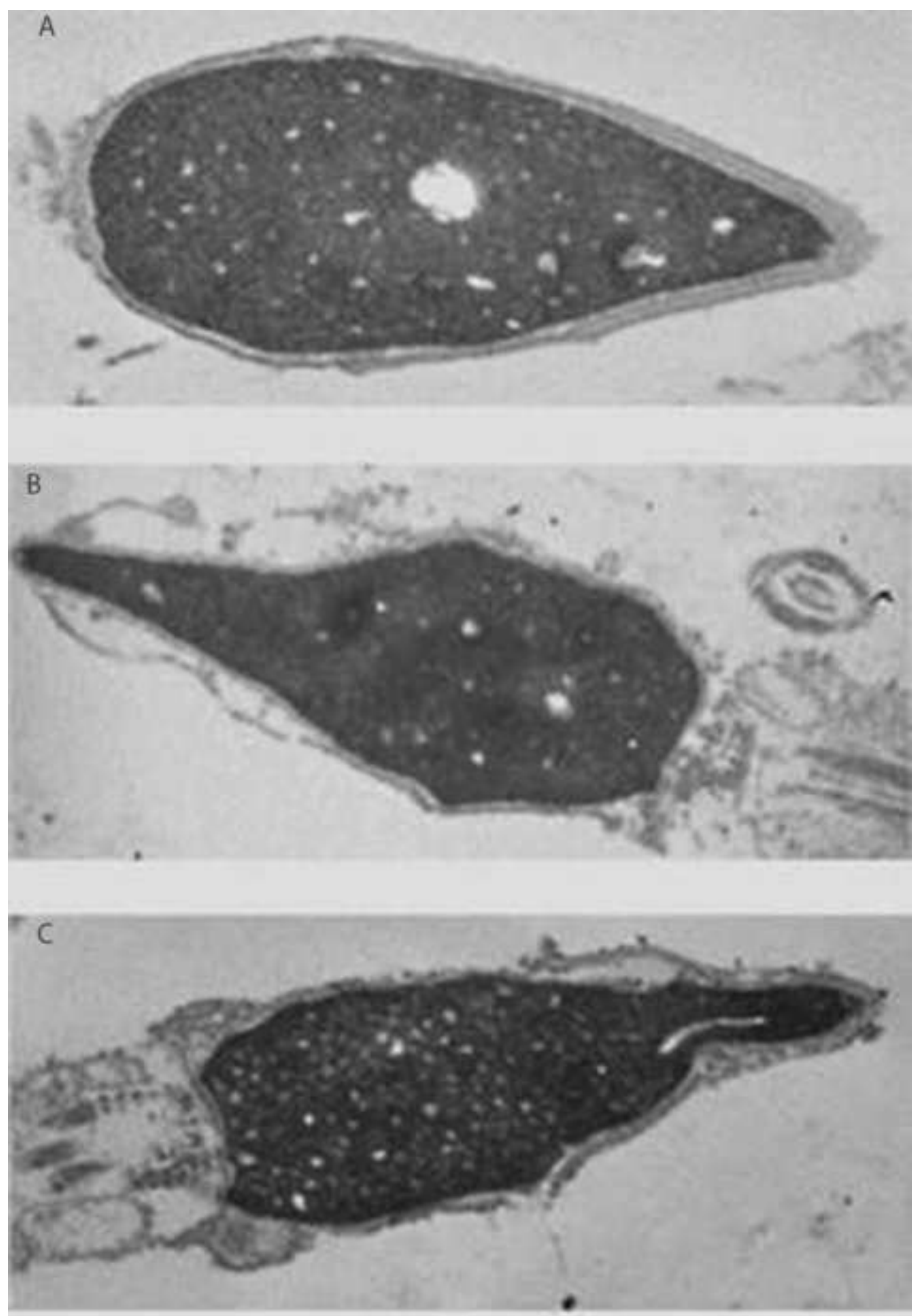